\title{
AUTOMATIC EMOTION INDUCTION AND ASSESSMENT FRAMEWORK Enhancing User Interfaces by Interperting Users Multimodal Biosignals
}

\author{
Jorge Teixeira, Vasco Vinhas, Luís Paulo Reis and Eugénio Oliveira \\ FEUP - Faculdade de Engenharia da Universidade do Porto, Rua Dr. Roberto Frias s/n, Porto, Portugal \\ DEI - Departamento de Engenharia Informática, Rua Dr. Roberto Frias s/n, Porto, Portugal \\ LIACC - Laboratório de Inteligência Artificial e Ciência de Computadores, Rua do Campo Alegre 823, Porto, Portugal \\ jtf@fe.up.pt,vvm@fe.up.pt,lpreis@fe.up.pt,eco@fe.up.pt
}

\begin{abstract}
Keywords: $\quad$ Biosignals, Emotions, Classification, Multimedia, Clustering.
Abstract: Emotion's definition, identification, systematic induction and efficient and reliable classification have been themes to which several complementary knowledge areas such as psychology, medicine and computer science have been dedicating serious investments. This project consists in developing an automatic tool for emotion assessment based on a dynamic biometric data acquisition set as galvanic skin response and electroencephalography are practical examples. The output of standard emotional induction methods is the support for classification based on data analysis and processing. The conducted experimental sessions, alongside with the developed support tools, allowed the extraction on conclusions such as the capability of effectively performing automatic classification of the subject's predominant emotional state. Self assessment interviews validated the developed tool's success rate of approximately $75 \%$. It was also experimentally strongly suggested that female subjects are emotionally more active and easily induced than males.
\end{abstract}

\section{INTRODUCTION}

Emotions play an important role in all human activities, from the trivial to the most complex ones. This significance is translated both in terms of reality perception and even in the cognitive decision process. Meanwhile, computers have gained such a relevant presence in the modern society that they have been introduced in almost every aspect of it, enhancing the magnitude of ubiquitous computing.

Having these two realities in mind - the importance of emotional states and the necessity of daily interaction with multiple devices - merging them would be a great improvement. By providing the distributed computer systems with the perception of their users' emotions, the applications would be able to adjust their interface, promote and suggest functionalities accordingly. It is believed that this approach would increase the global system's transparency and efficiency as its dynamism would follow in the encounter to user's intentions and temper.

Alongside the ubiquitous computing, multimedia contents are becoming constantly more complex and seemlier to reality, enabling a greater action immersion sensation, the primitive absolute need of achieving a perfect match between audiovisual contents and the audience desires is still present and constitutes the main key to the industry success.

The alliance between the multimedia contents choice possibility that enables the audience to individually presence what desires and accurate emotional states detection systems leads to subconscious individual interaction between the audience and the multimedia control system, potentiating the perfect match between content and individual audience desires.

This study illustrates a proposal for an application that enables automatic emotional state assessment using minimal invasive solutions.

The rest of the paper is organized as follows: in the next section the current state of the art is presented; in section 3 a project description is given; in section 4 the study's results are depicted and, consequently, the project's conclusions are listed and future work areas are identified in the final section. 


\section{STATE OF THE ART}

An emotional state can be defined as a collection of responses triggered by different parts of the body or the brain through both neural and hormonal networks (Damásio, 1998). Experiments conducted with patients with brain lesions in specific areas led to the conclusion that their social behaviour was highly affective, together with the emotional responses. It is unequivocal to state that emotions are essential for humans, as they play a vital role in their everyday life: in perception, judgment and action processes (Damásio, 1994).

Due to the complexity of human emotions, their analysis is often based on the identification of distinct basic emotional states so that the analysis and processing is simplified. For this project, three emotional states will be studied: joy, sadness and a neutral emotional state.

In order to analyse biometric data that contains mainly positive and negative emotional states, it is essential to create and define an experimental environment that is able to induce a subject in a specific and controlled emotional state. Nowadays, it is common to use an actor as one possible approach to human beings emotions' simulation (Chanel et al., 2005). As the actor predicts specific emotions, outside aspects as facial expression or voice change accordingly. However, the physiological responses will not suffer any variations, which lead to one of the biggest disadvantages of this approach, as the gathered biometric information does not represent the real emotional state of the actor.

An alternative method, adopted in this study, is the use of multimedia stimuli (Chanel et al., 2005). These stimuli contain a variety of contents such as music, videos, text and images. The main advantage of this method resides in the strong correlation between the induced emotional states and the physiological responses, as the emotions are no longer simulated.

The electroencephalograph used on this project was the NeurobitLite and is composed by 3 electrodes, one functioning as and active one and the other two as references, using a monopolar method strategy.

\section{PROJECT DESCRIPTION}

The development of an automatic tool that able to determine the emotional state of a subject through EEG biometric information was based on data analysis' methods. These methods included the decimation; the weighted average; spikes' removal; and clusters for the final emotion's assessment.
The implementation of the weighted average technique culminated with the enunciation of a hypothesis concerning the behaviour of the electrical brain waves when subjects are emotionally induced. The hypothesis follows a specific temporal distribution and a pattern that was observed in the majority of the experimental sessions. Figure 3 represents the evolution of Beta and Gamma amplitudes' over the entire experimental session. This behaviour is considered as the pattern behaviour for high frequency brain waves (Teixeira, Vinhas, 2008).

The enunciated expected behaviour represents a three step chart with each step having the duration of two minutes. This data treatment had in mind the three steps took into account to IAPS session management - three sets of twenty pictures lasting for two minutes with a grading emotional effect from joy to sadness passing through an intermediate neutral state.

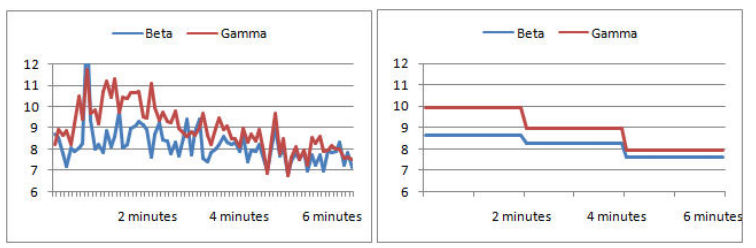

Figure 1: Expected behaviour for high frequency waves

In what concerns to cluster analysis, the emotional induction results in an amplitude's variation according to a specific emotional state. Having these concepts in mind, and based on the pattern-behaviour for the EEG data previously described, three distinct groups of data were created based on the brain waves' mean amplitude. Each of these three groups have one specific centroide, a point that is used as a reference for the neighbours of the same cluster.

The emotional state classification, performed by the EAT, is based on the predominant emotional state of the subject, as previously described. In order to evaluate the success rate of the EAT classification, at the end of each experimental session, selfassessment interviews were performed to subjects. The main objective of these interviews was to attain information concerning the predominant emotional state, so that it could be later compared with the results obtained from the EAT analysis. Besides this fact, other important aspects like the apreciation of the presented visual stimuli sequence, the environment conditions and any disturbs during the experimental session have been collected. 
These interviews constituted an essential database for project results' validation purposes.

The emotional induction is divided in three distinct and perfectly defined stages: joy, sadness and a neutral state. This allows determining which of the stages is more efficient during the whole experimental session or has stronger and more coherent effect on the subject. This hypothesis is the base of the concept for the EAT, so that it is able to determine, based on the gathered EEG biometric data, the predominant emotional state of the subject.

Based on the statistical analysis, each of the steps is directly associated with one of the clusters, so that there are three distinct clusters per experimental session. The clusters' analysis is based on the centroides' values and the number of samples, so that the global organization of the samples is different for each emotional state.

Aside with these characteristics, the emotions' assessment was performed for both Beta and Gamma brain waves, since high frequency brain waves are believed to have the most noteworthy changes due to emotional states transition (Teixeira, Vinhas et al, 2008).

\section{RESULTS}

From the experimental sessions conducted two different kind of results were achieved: the first belongs to the visual analysis performed and is based on the pattern-behaviour defined for the high frequency brain waves; the other concerns to the results obtained from the application of the EAT to the biomatric data captured during the experimental sessions.

The enunciated hypothesis was proved and validated by the application of the data analysis' of the experimental sessions. Gathered biometric data showed a high degree of similarity between the behaviour of the high frequency brain waves and the hypothesis previously stated when the predominant emotional state of the subject is coincident.

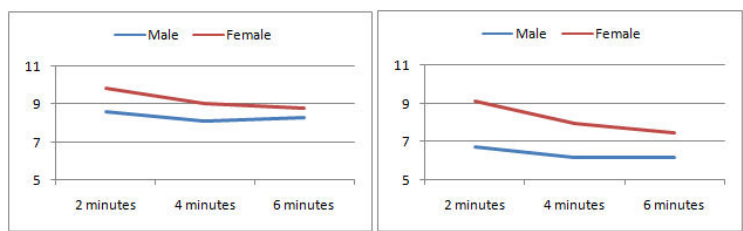

Figure 2: Beta wave (a) and Gamma wave (b) comparison for men and women

Apart from these results, the comparasion of men and women brain wave behaviour, presented in
Figure 4, indicates differences in amplitude directly related with the sex of the subject. The presented charts were based on the average amplitude of all subjects, male and female seperatly, for the three distinct session stages.

Through this ilustration, it is shown a slighty decreasing variation of the average amplitude along the entire experimental session, which proves the pattern-behaviour previously described. Together with these results, also an amplitude diference between male and female waves is presented during the session, with a higher amplitude for the female behaviour.

Based on the previously described results, as well as the statistical analysis, the EAT was able to follow the initial hypothesis. The emotional state decision taken by the aplicaiton is based on the clusters' length, centroides' value and the respective high frequency brain wave.

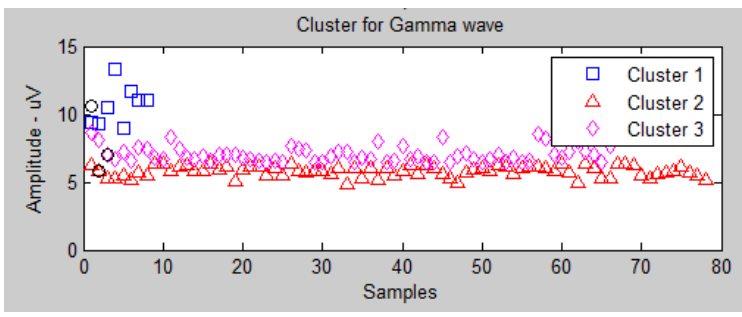

Figure 3: Cluster analysis for the Gamma wave

Figure 5 represents the cluster analysis of one experimental session for the Gamma wave. These results indicate a clear majority of data associated with the lowest centroide value, and a small density of data near the high value centroide. Based on this approach, the emotional state classification is based on the clusters' length and its degree of correlation between each others.

For this specific project, there were studied two different emotional states plus the neutral one, so that three clusters were adopted for the statistical analysis. The integration of this tool in a project with a bigger scope is suitable and advantageous, since the number of clusters is dependent on the number of emotional states to analyse and the specific multimedia content used for the emotional induction.

Besides the emotion assessment functionality, this tool also integrates some important features for data analysis as: plotting the original biometric data gathered from the EEG; calculate the weighted means directly from the original signal in intervals of 5, 10 and 20 seconds, defined by the user; activate or deactivate the spikes removal technique, affecting and improving the assessment results for more unstable experimental sessions. 


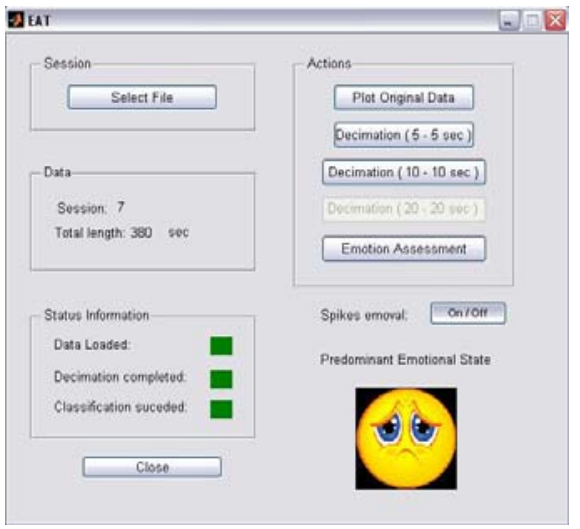

Figure 4: EAT running screenshot

Figure 6 represents an EAT running screenshot, where the final conclusion led to a predominant emotional state of sadness after a decimation of 20 seconds of the load of raw session data file. The performance attained through the application of the EAT is directly related with the success rate of the emotional assessment and is a determinant factor for the verification and validation of this tool for future work. Accordingly to Table 1, where the confusion table is presented, the final rate of success is $74 \%$ and all the failures of the EAT are related with the sadness emotional state, with low values for the brain waves amplitude.

\begin{tabular}{|c|c|c|c|c|}
\cline { 3 - 5 } \multicolumn{2}{c|}{} & \multicolumn{3}{c|}{ EAT } \\
\cline { 3 - 5 } \multicolumn{2}{c|}{} & Joy & Neutral & Sadness \\
\hline \multirow{己}{*}{$\cdot \overrightarrow{0}$} & Joy & $\mathbf{1 1 \%}$ & $0 \%$ & $11 \%$ \\
\cline { 2 - 5 } & Neutral & $0 \%$ & $\mathbf{0 \%}$ & $0 \%$ \\
\cline { 2 - 5 } & Sadness & $0 \%$ & $16 \%$ & $\mathbf{6 3 \%}$ \\
\hline
\end{tabular}

Table 1: Confusion Table

The application of the EAT for the automatic assessment was, for one of the experimental sessions, able to determine the correct predominant emotional state, which wasn't possible through the empirical visual inspection analysis of the biometric data after processing it. This indicates that the $16 \%$ of failure of the EAT are related to a discrepancy between the analysis of the Beta and Gamma brain waves' behaviour.

\section{CONCLUSION}

The execution of twenty eight experimental sessions based on a predefined induction method resulted on a vast collection of biometric data. The initial enunciated hypothesis was validated and the majority of the subjects included in this project have reacted in a similar way to the multimedia content presented. Starting with light, enjoyable contents and finishing with sad ones, it was able to conclude that the average amplitude of the high frequency brain waves decreased along the entire session based on the emotional state induced on the subject. Two different emotional states - joy and sadness - plus a neutral one led to the use of three clusters, characterized by its centroides' value, the number of samples included and the degree of correlation between them. EAT was able to classify the predominant emotional state, out of three, with an accuracy of almost $75 \%$.

One shall consider system adaptations in order to accommodate psychiatric diagnosis and treatment procedures, either by simple emotional state assessment or by complementing this feature with audiovisual adequate contents. Videogame related entertainment industry is also a potential target with the introduction of emotional state information as an extra variable for game play enhancement. There have been identified some future work such as the necessity of performing the referred assessment in real-time, following a sliding window approach for containing some historical and contextual information. Once this enhancement becomes real, it would be interesting to expand the number of emotional states detectable by the application, such as anger and excitement.

As a final remark, one shall state that the presented study achieved to develop an automatic tool for basic emotional states detection with high rates of success based on pre-defined stable experimental methodologies of emotion induction and data processing and validation. The used hardware solutions are believed to be minimal invasive and are not costly which enables its vast application at a larger scale.

\section{REFERENCES}

Chanel, G., et al. 2005. Emotion Assessment: Arousal Evaluation using EEG's and Peripheral Physiological Signals. University of Geneva, Switzerland: Computer Science Department, pp 530-537, vol 4105/2006.

Damásio, A. R. 1994. Descartes error: Emotion, reason and human brain. Europa-América.

Damásio, A. R. 1998. Emotions and the Human Brain. Iowa, USA: Department of Neurology.

Teixeira, J., Vinhas, V. et al. 2008. Multichannel Emotion Assessment Framework: Gender and High-Frequency Electroencephalography as Key-Factors. ICINCO $2008,5^{\text {th }}$ International Conference on Informatics in Control, Automation and Robotics. 